University of Nebraska - Lincoln

DigitalCommons@University of Nebraska - Lincoln

8-22-2002

\title{
Bulk and interfacial properties of quadrupolar fluids
}

V.B. Warshavsky

University of Nebraska-Lincoln

Xiao Cheng Zeng

University of Nebraska-Lincoln, xzeng1@unl.edu

Follow this and additional works at: https://digitalcommons.unl.edu/chemzeng

Part of the Chemistry Commons

Warshavsky, V.B. and Zeng, Xiao Cheng, "Bulk and interfacial properties of quadrupolar fluids" (2002).

Xiao Cheng Zeng Publications. 40.

https://digitalcommons.unl.edu/chemzeng/40

This Article is brought to you for free and open access by the Published Research - Department of Chemistry at DigitalCommons@University of Nebraska - Lincoln. It has been accepted for inclusion in Xiao Cheng Zeng Publications by an authorized administrator of DigitalCommons@University of Nebraska - Lincoln. 


\title{
Bulk and interfacial properties of quadrupolar fluids
}

\author{
V. B. Warshavsky and X. C. Zeng \\ Department of Chemistry, University of Nebraska-Lincoln, Lincoln, Nebraska 68588
}

(Received 5 April 2002; accepted 31 May 2002)

\begin{abstract}
We extend Teixeira and Telo da Gama's density-functional approach [J. Phys.: Condens. Matter 3, 111 (1991)] to study the vapor-liquid phase equilibria and planar interfacial properties of quadrupolar fluids. The density profile, surface ordering, surface polarization, and surface tension of quadrupolar fluids are evaluated. Particular attention is given to the temperature and quadrupole-strength dependence. It is found that the interfacial molecular ordering can arise entirely due to the quadrupole-quadrupole interaction. It is also found that the bulk-phase properties of the quadrupolar fluids satisfy the law of correspondence states. The reduced surface potential and surface tension can be well correlated by power laws of the scaled temperature $\tau \equiv 1-T / T_{c}$, where $T_{c}$ is the critical temperature of the fluids. (C) 2002 American Institute of Physics.
\end{abstract}

[DOI: $10.1063 / 1.1495841]$

\section{INTRODUCTION}

Quadrupolar molecules such as $\mathrm{O}_{2}, \mathrm{~N}_{2}$, and $\mathrm{CO}_{2}$ abound in nature ${ }^{1}$ and thus quadrupolar fluids are of both fundamental and practical importance. In the past two decades many model fluids with embedded point quadrupole have been studied using liquid-state theories and molecular simulations. These include, for example, quadrupolar hardsphere fluids, quadrupolar Lennard-Jones (LJ) fluids, quadrupolar hard diatomic molecular fluids, and ideal (point) quadrupolar two-center LJ fluids. Special attention has been given to the bulk-phase properties such as the vapor-liquid phase equilibria, ${ }^{2-7}$ thermodynamic free energy, the equation of states, ${ }^{8,9}$ and liquid structures. ${ }^{10-12}$ Bulk-phase properties of some more sophisticated nonspherical molecular fluids such as quadrupolar linear-Kihara fluids, quadrupolar harddumbbell fluids, and quadrupolar hard Gaussian-overlap fluids have also been investigated. ${ }^{13-17}$

Compared to the bulk-phase properties, less effort has been dedicated to the study of interfacial properties of quadrupolar fluids. A better understanding of the interfacial properties of quadrupolar fluids can be useful, for example, to the study of heterogeneous droplet formation containing quadrupolar component. Haile, Gray, and Gubbins ${ }^{18}$ were apparently the first to investigate interfacial properties of quadrupolar site-site LJ fluids. They developed a thermodynamic perturbation theory based on the Padé approximation to calculate the Fowler model (abrupt vapor-liquid transition) surface tension of the liquids. Later, Thompson, Gubbins, and Haile ${ }^{19}$ reported an improved perturbation theory using the Mayer $f$ function as the expansion functional. This "f-expansion" theory predicts orientational ordering at the planar liquid-vapor surface due to quadrupolar forces. Thompson et al. found that when the strength of the quadrupole moment is small the molecules tend to orient themselves perpendicular to the interface on the liquid side. However, when the strength of the quadrupole moment is moderately strong the molecules tend to orient parallel to the surface. This prediction is in qualitative agreement with the molecular dynamic (MD) simulation. ${ }^{20}$

Since the eighties, density functional theories (DFT) have been widely used for the study of interfacial properties of molecular fluids. The DFT allows one to calculate both structural (density and orientational profiles) and thermodynamical (surface tension) properties in a self-consistent fashion. An important input required in the DFT is the correlation function which can be obtained either from the integral equation theories ${ }^{21-23}$ or by using some approximations. The modified mean field approximation (MMF) proposed by Teixeira and Telo da Gama ${ }^{24}$ is a very simple choice beyond the mean field, for which the radial distribution function is approximated by a low-density form. For dipolar fluids, the MMF-DFT predicts that stronger dipole moments yield higher surface tension and the interfacial molecular ordering can be induced solely by the dipolar forces. The MMF-DFT has been extended to treat various molecular systems such as binary dipolar fluids ${ }^{27}$ and TIP4P model water, ${ }^{28}$ and has also been generalized to investigate the vapor-phase nucleation of Stockmayer fluids, ${ }^{29}$ the electric field effects on the interface of dipolar fluids, ${ }^{30}$ and the Tolman length of dipolar fluids. ${ }^{31}$ Here we extend the MMF-DFT to study the vapor-liquid phase equilibria, surface ordering, surface polarization and surface tension of a model quadrupolar fluid. Note that several studies of the interfacial properties of quadrupolar fluids using DFT have been reported in the literatures. ${ }^{28,32}$ However, the prediction of the interfacial molecular ordering thus far was entirely based on the dipole-quadrupole interaction. To our knowledge, interfacial molecular ordering due to pure quadrupole-quadrupole interaction has not been reported.

Teixeira and Telo da Gama, ${ }^{24}$ within the framework of their MMF-DFT, examined the interfacial ordering of the point quadrupolar fluid. In their perturbative scheme the grand canonical potential was expanded over the powers of the quadrupole-quadrupole interaction. They only considered the first-order term, however, and as a result, they did not find any orientational ordering at the vapor-liquid interface. Nonetheless, they pointed out that quadrupole-induced 
interfacial ordering may arise if quadrupole-quadrupole terms to the second order were considered. Here, we confirm their prediction, as will be shown in next sections.

The rest of the paper is organized as follows: In Sec. II we extend the MMF-DFT to treat a model quadrupolar fluid. A set of equations for evaluating the density and orientation profiles is derived. In Sec. III we present vapor-liquid coexistence curves, density and order parameter profiles, surface polarization and surface tension as well as their temperature and the strength of quadrupole moment dependence and related scaling relations. The conclusion is given in Sec. IV.

\section{DENSITY FUNCTIONAL THEORY FOR QUADRUPOLAR FLUIDS}

In this section we extend the MMF density functional theory ${ }^{24,25,29}$ to a model quadrupolar fluid system. We consider a single-component fluid composed of linear (axial) molecules with an electric quadrupole moment. In the bodyfixed spatial coordinates with the molecular axis as the $z^{\prime}$-axis the tensor of quadrupole moment has only one nonzero component, $Q_{z^{\prime} z^{\prime}}=\sum_{i} q_{i} z_{i}^{\prime 2}$, where $q_{i}$ is the charge on the $i$-site and $z_{i}^{\prime}$ its position relative to the center of the molecule. Hereafter we denote $Q_{z^{\prime} z^{\prime}}$ as $Q$. In general, the number density of molecules $\rho(\mathbf{r}, \omega)$ depends on both the spatial $\mathbf{r}=(x, y, z)$ and angular $\omega=(\theta, \phi)$ coordinates of the molecules. Here, $\omega$ describes the relative orientation of the molecular axis with respect to the space-fixed coordinate. The orientation profile $\hat{f}(\mathbf{r}, \omega)$ can be defined by

$$
\rho(\mathbf{r}, \omega)=\rho(\mathbf{r}) \hat{f}(\mathbf{r}, \omega),
$$

where $\rho(\mathbf{r})$ is the density profile integrated over all the orientations; thus, $\int d \omega \hat{f}(\mathbf{r}, \omega)=1$. The pairwise intermolecular potential $u\left(\mathbf{r}_{1}, \mathbf{r}_{2}, \omega_{1}, \omega_{2}\right)$ can be written as a sum of pairwise intermolecular potential of the reference system $u_{\text {ref }}\left(\mathbf{r}_{1}, \mathbf{r}_{2}, \omega_{1}, \omega_{2}\right)$ and perturbation potential $u_{\text {per }}\left(\mathbf{r}_{1}, \mathbf{r}_{2}, \omega_{1}, \omega_{2}\right)$, i.e., $u\left(\mathbf{r}_{1}, \mathbf{r}_{2}, \omega_{1}, \omega_{2}\right)$ $=u_{\text {ref }}\left(\mathbf{r}_{1}, \mathbf{r}_{2}, \omega_{1}, \omega_{2}\right)+u_{\text {per }}\left(\mathbf{r}_{1}, \mathbf{r}_{2}, \omega_{1}, \omega_{2}\right)$, where $\mathbf{r}_{1}$ and $\mathbf{r}_{2}$ denote the spatial coordinates, and $\omega_{1}$ and $\omega_{2}$ the angular coordinates of two molecules.

In the framework of the MMF density-functional approach, ${ }^{24,25,29}$ the grand canonical potential in the absence of external field is given by

$$
\begin{aligned}
& \Omega[\rho(\mathbf{r}, \omega)] \\
& =\int d \mathbf{r} f_{\mathrm{ref}}(\rho(\mathbf{r}))-\iint d \mathbf{r} d \omega \mu \rho(\mathbf{r}, \omega) \\
& \quad+(1 / 2 \beta) \iiint \int d \mathbf{r}_{1} d \mathbf{r}_{2} d \omega_{1} d \omega_{2} \rho\left(\mathbf{r}_{1}, \omega_{1}\right) \\
& \quad \times \rho\left(\mathbf{r}_{2}, \omega_{2}\right) e^{-\beta u_{\mathrm{ref}}\left(\mathbf{r}_{12}, \omega_{1}, \omega_{2}\right)}\left[1-e^{-\beta u_{\mathrm{per}}\left(\mathbf{r}_{12}, \omega_{1}, \omega_{2}\right)}\right],
\end{aligned}
$$

where $\mathbf{r}_{12}=\mathbf{r}_{2}-\mathbf{r}_{1}, \mu$ is the chemical potential, and $\beta$ $=1 / k_{B} T$ ( $T$ is the temperature and $k_{B}$ the Boltzmann constant). The last term on the right-hand side of Eq. (2) is the interaction contribution due to the long-range part of the pair potential between the spherical particles with embedded point quadrupole. The Helmholtz free energy density of the isotropic reference system can be written as

$$
f_{\text {ref }}(\rho(\mathbf{r}))=f_{\text {hs }}(\rho(\mathbf{r}))+k_{B} T \rho(\mathbf{r})\langle\ln [4 \pi \hat{f}(\mathbf{r}, \omega)]\rangle,
$$

where $\langle\ldots\rangle=\int d \omega \ldots \hat{f}(\mathbf{r}, \omega)$. The first term on the right-hand side of Eq. (3) is the free energy density of the hard-sphere system, which can be accurately calculated using the Carnahan-Starling formula; ${ }^{33}$ the second term is due to the loss of the entropy caused by the orientational molecular ordering.

Here, we consider a model quadrupolar fluid system with the reference potential given by

$$
u_{\mathrm{hs}}\left(r_{12}\right)= \begin{cases}+\infty, & r_{12} \leqslant d \\ 0, & r_{12}>d\end{cases}
$$

and the perturbation potential given by

$u_{\mathrm{per}}\left(\mathbf{r}_{12}, \omega_{1}, \omega_{2}\right)=\left[u_{00}\left(r_{12}\right)+u_{q q}\left(\mathbf{r}_{12}, \omega_{1}, \omega_{2}\right)\right] H\left(r_{12}-d\right)$

In Eqs. (4) and (5), $d$ is the hard-sphere diameter, $H(r)$ is the Heaviside step function, and the isotropic part of the intermolecular interaction $u_{00}$ is chosen as the augmented Sutherland potential ${ }^{34,35}$ such as

$$
u_{00}\left(r_{12}\right)=-4 \epsilon\left(d / r_{12}\right)^{6} \text {, }
$$

where $\epsilon$ is the energy parameter of the Sutherland potential. The contribution $u_{q q}\left(\mathbf{r}_{12}, \omega_{1}, \omega_{2}\right)$ is the potential function between two linear quadrupoles, ${ }^{36}$

$$
\begin{aligned}
u_{q q}\left(\mathbf{r}_{12}, \omega_{1}, \omega_{2}\right) \\
=\frac{3}{4} \frac{Q^{2}}{r_{12}^{5}}\left(1-5 c_{1}^{2}-5 c_{2}^{2}+2 c_{12}^{2}+35 c_{1}^{2} c_{2}^{2}-20 c_{1} c_{2} c_{12}\right),
\end{aligned}
$$

where $c_{i}=\mathbf{n}_{i} \cdot \mathbf{n}(i=1,2)$ and $c_{12}=\mathbf{n}_{1} \cdot \mathbf{n}_{2} ; \mathbf{n}_{1}$ and $\mathbf{n}_{2}$ is the unit vector along the molecular axes of molecule 1 and 2 , respectively, and $\mathbf{n}=\mathbf{r}_{12} / r_{12}$ is the unit intermolecular radius vector.

The fluid system is considered to be an infinite slab with the Cartesian axis $z$ normal to the slab surfaces. The planar vapor-liquid interface is in the $x-y$ plane and in parallel with the slab surfaces. Thus, the system is inhomogeneous only in the $z$ direction so that both the density profile $\rho(z)$ and the orientation distribution function $\hat{f}(z, \omega)$ are dependent only on the spatial variable $z$ but not on $x$ and $y$. The orientation distribution function $\hat{f}(z, \omega)$ can be conveniently written as a sum of the isotropic term $1 / 4 \pi$ and a small correction term $\Delta \hat{f}(z, \omega)(\Delta \hat{f} / \hat{f} \ll 1)$ due to anisotropy arising from the weak quadrupole-quadrupole interaction, that is

$$
\hat{f}(z, \omega)=1 / 4 \pi+\Delta \hat{f}(z, \omega) \text {. }
$$

With Eqs. (1), (3), and (8), Eq. (2) can be transformed into the following expression: 


$$
\begin{aligned}
& \Omega[\rho(z, \omega)] / A \\
& =\int_{-\infty}^{\infty} d z\left\{f_{\mathrm{hs}}(\rho(z))+2 \pi k_{B} T \rho(z) \int d \omega|\Delta \hat{f}(z, \omega)|^{2}\right\} \\
& \quad-\int_{-\infty}^{\infty} d z \mu \rho(z)+\frac{1}{2} \int_{-\infty}^{\infty} \int_{-\infty}^{\infty} d z_{1} d z_{2} \rho\left(z_{1}\right) \rho\left(z_{2}\right) \\
& \quad \times \iint d \omega_{1} d \omega_{2} \hat{f}\left(z_{1}, \omega_{1}\right) \hat{f}\left(z_{2}, \omega_{2}\right) \phi_{\mathrm{eff}}\left(z_{12}, \omega_{1}, \omega_{2}\right),
\end{aligned}
$$

where $A$ is the area of the planar vapor-liquid interface, $z_{12}=z_{2}-z_{1}$, and the effective potential $\phi_{\text {eff }}$ is given in the Appendix. Note that in deriving Eq. (9) we expanded the exponential term in Eq. (2) up to the second order in $\beta u_{\text {per }}$ rather than to the first order (see the Appendix for more details). This is based on the fact ${ }^{24}$ that no orientational ordering at the interface can be observed if only the quadrupole-quadrupole interaction term $\left(\beta u_{\text {per }}\right.$ term) to the first order is considered.

Since the system under consideration is invariant in the $x-y$ plane, the anisotropic part of the orientation distribution function $\Delta \hat{f}(z, \omega)$ is independent of the axial angle $\varphi$. As a result, $\Delta \hat{f}(z, \omega)$ can be expanded in terms of the Legendre polynomials $P_{k}(\cos \theta)$ with the coefficients of the expansion $\left\{\eta_{k}(z)\right\}$ serving as the orientation order parameters, that is

$$
\begin{aligned}
& \Delta \hat{f}(z, \omega)=\frac{1}{4 \pi} \sum_{k=1}^{\infty}(2 k+1) \eta_{k}(z) P_{k}(\cos \theta), \\
& \eta_{k}(z)=\left\langle P_{k}\right\rangle=\int d \omega P_{k}(\cos \theta) \hat{f}(z, \omega) \quad(k=1,2, \ldots) .
\end{aligned}
$$

Substituting Eqs. (8) and (10) into Eq. (9), the grand canonical potential per unit area becomes

$$
\begin{aligned}
& \Omega\left[\rho(z),\left\{\eta_{k}(z)\right\}\right] / A \\
& =\int_{-\infty}^{\infty} d z\left\{f_{\mathrm{hs}}(\rho(z))+\frac{1}{2 \beta} \rho(z) \sum_{k=1}^{\infty}(2 k+1) \eta_{k}^{2}(z)\right\} \\
& \quad-\int_{-\infty}^{\infty} d z \mu \rho(z)+\frac{1}{2} \int_{-\infty}^{\infty} \int_{-\infty}^{\infty} d z_{1} d z_{2} \\
& \quad \times \rho\left(z_{1}\right) \rho\left(z_{2}\right) \psi\left(z_{1}, z_{2}\right),
\end{aligned}
$$

where

$$
\begin{aligned}
\psi\left(z_{1}, z_{2}\right)= & \phi_{00}\left(z_{12}\right)+\phi_{22}\left(z_{12}\right) \eta_{2} \eta_{2}^{\prime}+\phi_{44}\left(z_{12}\right) \eta_{4} \eta_{4}^{\prime} \\
& +\phi_{02}\left(z_{12}\right)\left(\eta_{2}+\eta_{2}^{\prime}\right)+\phi_{04}\left(z_{12}\right)\left(\eta_{4}+\eta_{4}^{\prime}\right) \\
& +\phi_{24}\left(z_{12}\right)\left(\eta_{2} \eta_{4}^{\prime}+\eta_{2}^{\prime} \eta_{4}\right)
\end{aligned}
$$

and where $\eta_{i}=\eta_{i}\left(z_{1}\right)$ and $\eta_{i}^{\prime}=\eta_{i}\left(z_{2}\right) \quad(i=2,4)$. The functions $\phi_{00}, \phi_{22}, \phi_{44}, \phi_{02}, \phi_{04}$, and $\phi_{24}$ are given in the Appendix.

Finally, the equilibrium density and orientation order parameter profiles can be determined from the variational principle applied to the grand canonical potential per unit area [Eq. (11)], that is

$$
\frac{\delta(\Omega / A)}{\delta \rho(z)}=0 \quad \text { and } \frac{\delta(\Omega / A)}{\delta \eta_{k}(z)}=0 \quad(k=1,2, \ldots) .
$$

The orientational order parameters are incorporated into the expression of $\Omega$ [Eq. (11)] at two places: a sum of squared order parameters (the orientational entropy) in the first integral, and in the expression of $\psi$. Since $\psi[$ Eq. (12)] depends only on the order parameter $\eta_{2}$ and $\eta_{4}$ the variation of $\Omega$ to $\eta_{k}(z)$ is equal to zero for all $k$ values except $k=2$ and 4 . Consequently, the variation yields three coupled equations,

$$
\begin{aligned}
& \beta \mu=\beta \mu_{\mathrm{hs}}\left(\rho\left(z_{1}\right)\right)+\frac{1}{2}\left[5 \eta_{2}^{2}\left(z_{1}\right)+9 \eta_{4}^{2}\left(z_{1}\right)\right] \\
& +\beta \int_{-\infty}^{\infty} d z_{2} \psi\left(z_{1}, z_{2}\right) \rho\left(z_{2}\right), \\
& \eta_{2}\left(z_{1}\right)=-\frac{\beta}{5} \int_{-\infty}^{\infty} d z_{2}\left[\phi_{22}\left(z_{12}\right) \eta_{2}\left(z_{2}\right)+\phi_{02}\left(z_{12}\right)\right. \\
& \left.+\phi_{24}\left(z_{12}\right) \eta_{4}\left(z_{2}\right)\right] \rho\left(z_{2}\right),
\end{aligned}
$$

and

$$
\begin{aligned}
\eta_{4}\left(z_{1}\right)= & -\frac{\beta}{9} \int_{-\infty}^{\infty} d z_{2}\left[\phi_{44}\left(z_{12}\right) \eta_{4}\left(z_{2}\right)+\phi_{04}\left(z_{12}\right)\right. \\
& \left.+\phi_{24}\left(z_{12}\right) \eta_{2}\left(z_{2}\right)\right] \rho\left(z_{2}\right) .
\end{aligned}
$$

In Eq. (14), $\mu_{\mathrm{hs}}(\rho(z))$ is the local chemical potential of the hard-sphere reference fluid.

We note that if those terms of the order $Q^{4}$ in the expression for coefficients $\phi_{00}, \phi_{22}, \phi_{44}, \phi_{02}, \phi_{04}, \phi_{24}$ (A16) are neglected, then the coefficients $\phi_{44}, \phi_{02}, \phi_{04}$, and $\phi_{24}$ will be just zero, and $\phi_{00}$ will be independent of $Q$; only $\phi_{22}$ will be proportional to $Q^{2}$. As such, Eqs. (15) and (16) will result in that $\eta_{2}=0$ and $\eta_{4}=0$. Clearly, this neglection of $Q^{4}$ terms explains the missing of any interfacial molecular ordering. ${ }^{24}$ Moreover, with the neglection of $Q^{4}$ terms, Eqs. (11) and (14) will lose entirely the dependence on $Q$, as will all the bulk-phase and interfacial properties of the quadrupole fluid. Therefore, to study the effects of the quadrupole strength on the bulk-phase and interfacial characteristics of the fluid, it is essential to include the $Q^{4}$ terms in all the coefficients $\phi_{i k}(i \leqslant k ; i, k=0,2,4)$ [see (Eq. A16)].

\section{NUMERICAL RESULTS AND DISCUSSION}

\section{A. Vapor-liquid coexistence}

In homogeneous bulk phases the density $\rho$ and the orientation order parameters $\eta_{2}$ and $\eta_{4}$ are uniform. Thus, the integrals in Eqs. (11), (14), (15), and (16) can be determined analytically. Since the integral $\int_{-\infty}^{\infty} d z_{2} \phi_{02}\left(z_{12}\right)$, $\int_{-\infty}^{\infty} d z_{2} \phi_{04}\left(z_{12}\right)$, and $\int_{-\infty}^{\infty} d z_{2} \phi_{24}\left(z_{12}\right)$ in Eqs. (15) and (16) vanish it follows that $\eta_{2}=0$ and $\eta_{4}=0$ in bulk phases. Equations (11) and (14) become accordingly

$\Omega=-p_{\mathrm{hs}}(\rho)+\frac{1}{2} \Psi(1 / T) \rho^{2} \quad$ and $\mu=\mu_{\mathrm{hs}}(\rho)-\Psi(1 / T) \rho$,

where $p_{\mathrm{hs}}=\rho \mu_{\mathrm{hs}}-f_{\mathrm{hs}}$ is the hard-sphere pressure and 


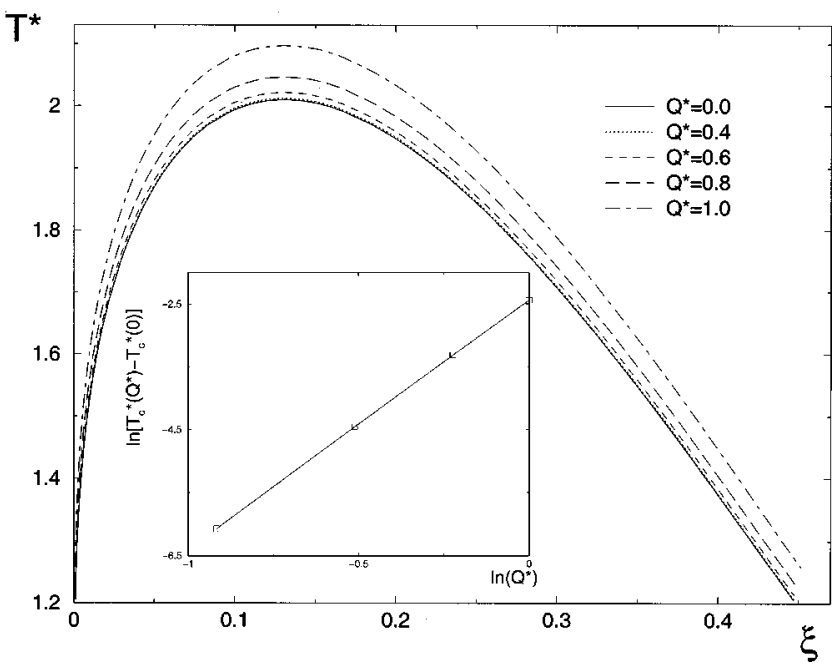

FIG. 1. Vapor-liquid coexistence densities for a quadrupolar fluid with various given $Q^{*}$. The inset shows the shift of the critical temperature $T_{c}^{*}\left(Q^{*}\right)-T_{c}^{*}(0)$ as a function of $Q^{*}$.

$$
\Psi(1 / T)=\frac{16}{3} \frac{\epsilon d^{3} \pi}{k_{B} T}\left(1+\frac{2}{3} \frac{\epsilon}{k_{B} T}+\frac{3}{20} \frac{Q^{4}}{\epsilon d^{10} k_{B} T}\right)
$$

is a measure of the attractive interaction energy of the quadrupolar fluid. The coexisting liquid and vapor density, $\rho_{l}$ and $\rho_{v}$, can be determined by solving the phase-equilibria equations, $\mu\left(\rho_{l}\right)=\mu\left(\rho_{v}\right)$ and $\Omega\left(\rho_{l}\right)=\Omega\left(\rho_{v}\right)$.

Figure 1 displays the vapor-liquid coexistence curves for a number of reduced quadrupole moment strength $Q^{*}$ $=Q /\left(\epsilon d^{5}\right)^{1 / 2}$ within $0 \leqslant Q^{*} \leqslant 1$. It can be seen that at a reduced temperature $T^{*}=k_{B} T / \epsilon$ increasing $Q^{*}$ decreases the coexistence vapor density $\xi_{v}\left(\xi \equiv \pi \rho d^{3} / 6\right.$ is the scaled density) and enlarges the coexistence liquid density $\xi_{l}$. The values of $\xi$ and $T^{*}$ at the critical point can be determined by solving the following equations:

$$
\partial^{3} \Omega /\left.\partial \xi^{3}\right|_{\xi_{c}}=0 \quad \text { and } \partial^{2} \Omega /\left.\partial \xi^{2}\right|_{\xi_{c}, T_{c}^{*}}=0 .
$$

Since the critical density $\xi_{c}$ is determined from the third derivative of the grand canonical potential, $\xi_{c}$ depends only on the reference hard-sphere system and has no $Q^{*}$ dependence. In fact, $\xi_{c}$ is a constant $\left(\xi_{c}=0.1304\right)$. The critical temperature $T_{c}^{*}$, however, is $Q^{*}$ dependent. It is found $T_{c}^{*}$ $=2.01,2.01,2.02,2.05$, and 2.10 for $Q^{*}=0.0,0.4,0.6,0.8$, and 1.0, respectively. In the inset of Fig. 1 we show that the difference in critical temperature from that of nonquadrupolar fluid, $T_{c}^{*}\left(Q^{*}\right)-T_{c}^{*}(0)$, follows very closely to the power law,

$$
T_{c}^{*}\left(Q^{*}\right)-T_{c}^{*}(0) \sim\left(Q^{*}\right)^{4} .
$$

It appears that this power law stems from the term with $Q^{4}$-dependence in Eq. (17), which is a mean-field result for weak $Q^{*}\left(Q^{*} \leqslant 1\right)$. Interestingly, Teixeira and Telo da Gama $^{24}$ have shown that for the dipolar fluids with reduced dipole moment $\mu_{0}^{*}=\mu_{0} /\left(\epsilon d^{3}\right)^{1 / 2}$ the difference in critical temperature follows a similar power law, ${ }^{24,26}$ that is, $T_{c}^{*}\left(\mu_{0}^{*}\right)-T_{c}^{*}(0) \sim\left(\mu_{0}^{*}\right)^{4}$. The relative change in $T_{c}^{*}$ for $\mu_{0}^{*}$ changing from 0 to 1 is $2.5 \%$, compared with $4.3 \%$ change for quadrupolar fluids with $Q^{*}$ changing from 0 to 1 .

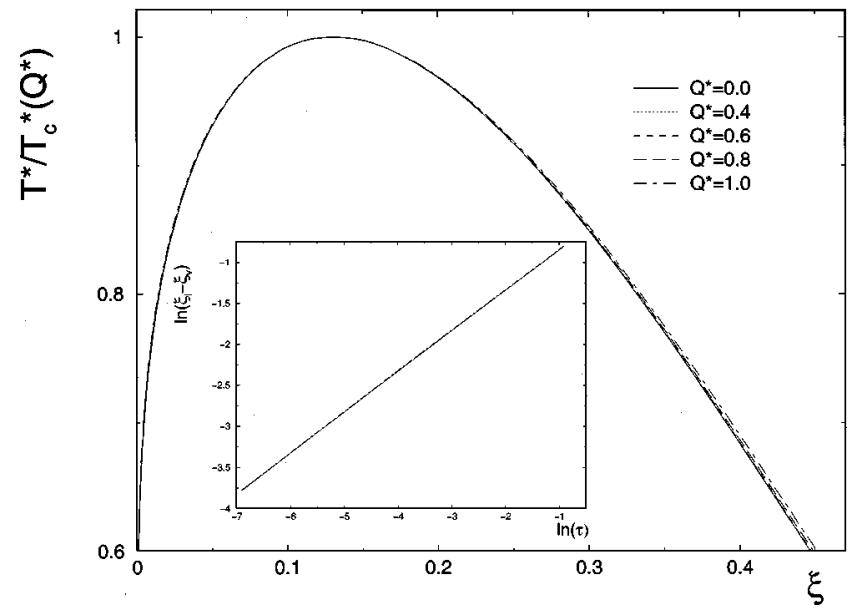

FIG. 2. The same as Fig. 1 except the temperature is rescaled based on the critical temperature. The inset shows that the difference between the coexisting liquid and vapor densities $\xi_{l}-\xi_{v}$ scales as $\tau^{1 / 2}$.

Several Monte Carlo (MC) simulations of the vaporliquid phase equilibria for the quadrupolar LJ fluids have been performed. ${ }^{2-4}$ Compared to the simulations, ${ }^{3,4}$ the present theory overestimates the value of critical temperature, although the model fluid is slightly different. The qualitative trend of $T_{c}^{*}$ as function of $Q^{*}$ is, however, the same. Smit et al. ${ }^{4}$ found that the effect of the reduced quadrupole moment $Q^{*}$ on $T_{c}^{*}$ is stronger than that of the reduced dipole moment $\mu_{0}^{*}$. For the pure $\mathrm{LJ}$ fluid ${ }^{37}$ it is found that $T_{c}^{*}$ $=1.31$, and for the dipolar $\mathrm{LJ}_{\text {fluid }}{ }^{38}\left(\mu_{0}^{*}=1\right), T_{c}^{*}=1.41$ which is about $7 \%$ higher. But for the quadrupolar LJ fluid ${ }^{4}$ $\left(Q^{*}=1.0\right), T_{c}^{*}=1.60$ which is about $22 \%$ higher.

We also examined whether the vapor-liquid coexistence densities (the binodal curve) satisfy the law of corresponding states. Figure 2 shows that all binodal curves for various given $Q^{*}$ can be almost collapsed onto a single curve by scaling the temperature with the $T_{c}^{*}$, that is, $T^{*}$ $\rightarrow T^{*} / T_{c}^{*}\left(Q^{*}\right)$. This result demonstrates that the law of corresponding state is indeed followed by the point quadrupolar fluids. The same conclusion has been drawn by Dubey and $\mathrm{O}^{\prime}$ Shea $^{39}$ from their MC simulation of quadrupolar LJ fluid. In the inset of Fig. 2 we show the scaling relationship for the difference between the coexisting liquid and vapor density $\xi_{l}-\xi_{v}$ and the small values of $\tau\left(\tau \equiv 1-T / T_{c}\right)$,

$$
\xi_{l}-\xi_{v} \sim \tau^{1 / 2} \text {. }
$$

The mean-field scaling exponent is 0.5 which is typically higher than the nonclassical exponent 0.325 expected close to the critical point. Note that the difference $\xi_{l}-\xi_{v}$ is nearly independent of the quadrupole moment strength $Q^{*}$ due to the law of corresponding state.

For purely dipolar fluids, Frodl and Dietrich ${ }^{25}$ have compared the results of the truncated MMF-DFT of Teixeira and Telo da Gama ${ }^{24}$ with the results of the full version of MMFDFT with the nonexpanded second exponential in Eq. (2). They showed that the truncated MMF-DFT introduces only quantitative but not qualitative errors which grow with the increasing of the dipole strength $\mu_{0}^{*}$. For instance, the critical temperature $T_{c}^{*}$ calculated from the truncated MMF-DFT is lower than that of the full version. At $\mu_{0}^{*}=1$ the difference 


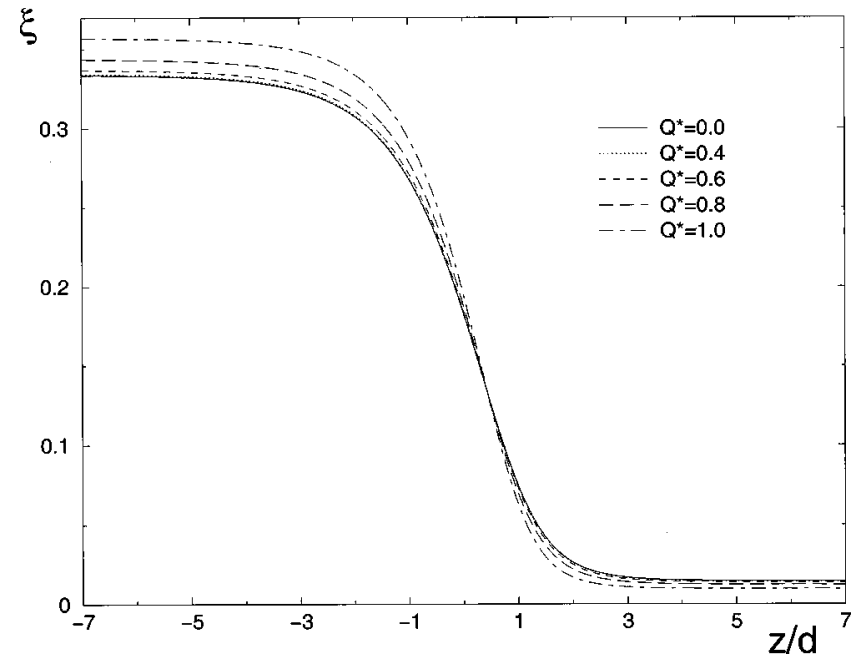

FIG. 3. The density profile $\xi(z)$ for various given $Q^{*}$ at $T^{*}=1.6$.

in $T_{c}^{*}$ between the truncated and full version MMF-DFT is about $10 \%$. The present approach, therefore, is suited best for weakly quadrupolar fluids $\left(Q^{*} \leqslant 1\right)$. For more strongly quadrupolar fluids $\left(Q^{*}>1\right)$ the truncation errors become large. Nonetheless we also calculated the phase coexistence densities and critical temperatures for some large values of $Q^{*}\left(1<Q^{*} \leqslant 2\right)$. Interestingly, we find that the power-law Eq. (20) (inset to Fig. 1) and the law of corresponding states (Fig. 2) no longer hold, however, the power law Eq. (21) still holds.

\section{B. Interfacial properties}

The density and orientation order-parameter profiles for the planar vapor-liquid interface are calculated by solving Eqs. (14)-(16) numerically using an iteration method. The initial input for the density profile is a step function for which the density is set to be the bulk liquid density $\xi_{l}$ for $z<0$ and the bulk vapor density $\xi_{v}$ for $z>0$. The initial input for both $\eta_{2}(z)$ and $\eta_{4}(z)$ is a constant zero. In Fig. 3 we show the scaled density profile $\xi(z) \equiv\left(\pi d^{3} / 6\right) \rho(z)$ for $Q^{*}$ ranging from 0 to 1 and at $T^{*}=1$.6. It is seen that as $Q^{*}$ increases the values of liquid-side density increase while the values of vapor-side density decrease, consistent with the result of vapor-liquid coexistence densities. Figure 4 shows the orientation order-parameter profile $\eta_{2}(z)$ for various given $Q^{*}$. Because $\eta_{4}(z)$ is much smaller in values than $\eta_{2}(z)$ at the same given $Q^{*}$ and $T^{*}, \eta_{4}(z)$ is not plotted. As defined above, $\eta_{2}(z)$ is related to the mean Legendre polynomial of the second order [Eq. (10)]. Thus, $\eta_{2}(z)<0$ indicates that the molecules tend to lie parallel to the interface on the liquid side, while on the vapor side the molecules tend to be perpendicular to the interface because of $\eta_{2}(z)>0$. The interfacial molecular behavior is similar to that of dipolar molecules at the vapor-liquid interface. ${ }^{21-24,26,40} \mathrm{We}$ have found that for $Q^{*} \leqslant 1, \eta_{2}(z)$ is always $<0.01$ in values, consistent with the weak anisotropy requirement to the distribution function $\hat{f}$ [see Eqs. (8) and (10)]. However, the relative increase of $\eta_{2}(z)$ for large $Q^{*}$ is substantial. We find that for

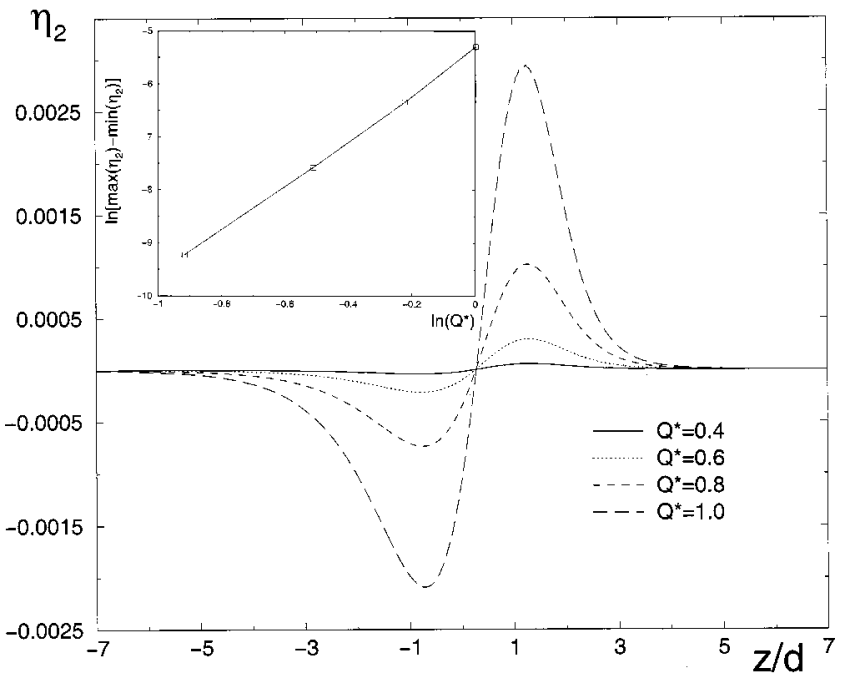

FIG. 4. The orientational order-parameter profile $\eta_{2}(z)$ for various given $Q^{*}$ and $T^{*}=1.6$. The inset shows the interfacial ordering scales as $\left(Q^{*}\right)^{4}$.

any given $T^{*}$ the function $\eta_{2}(z) /\left(Q^{*}\right)^{4}$ reduces to some limiting dimensionless function as $Q^{*}$ goes to zero.

It is convenient to characterize the magnitude of the interfacial ordering by using the difference between the maximum and minimum value of $\eta_{2}(z)$, i.e., $\max \eta_{2}(z)$ $-\min \eta_{2}(z)$. The inset of Fig. 4 shows this difference follows the power law,

$$
\max \eta_{2}(z)-\min \eta_{2}(z) \sim\left(Q^{*}\right)^{4} .
$$

Interfacial behavior of quadrupolar fluids has also been reported by other workers. ${ }^{19,32}$ Thompson et al. ${ }^{19}$ used the $f$-expansion theory to study the site-site LJ (SSLJ) plus quadrupole fluid at the reduced temperature $T^{*}=1.065$. Although the theory did not predict the dependence of the coexisting densities on $Q^{*}$, it does show that as $Q^{*}$ increases, the density profile becomes sharper, in accordance with the present result (see Fig. 3). Both their $f$-expansion theory ${ }^{19}$ and MD simulation ${ }^{20}$ showed that increasing $Q^{*}$ tends to alter interfacial ordering on the basis of nonquadrupolar SSLJ system. For large $Q^{*}$, molecules prefer to orient parallel to the interface on the liquid side and normal to the interface on the vapor side. This result is in accordance with ours (see Fig. 4). Chacón et al. ${ }^{32}$ applied the densityfunctional theory to study the systems governed by dispersion, overlap, dipole-dipole, dipole-quadrupole, quadrupole-quadrupole, and isotropic forces. Their calculated orientation order-parameter profile $\eta_{2}(z)$ is similar to ours (see Fig. 4). Because only the perturbation to the first order was used, the theory cannot predict any orientational order at the vapor-liquid interface for purely quadrupolar fluids. ${ }^{24}$

Figure 5 displays the density profiles for $Q^{*}=0.8$ at various scaled temperature $\tau$. We follow Rowlinson and Widom $^{41}$ to define the width of the interface $W_{G}=-\left(\rho_{l}\right.$ $\left.-\rho_{v}\right) /\left.(\partial \rho / \partial z)\right|_{z_{G}}$, where $z_{G}$ is the position of the Gibbs dividing surface, defined by the equation $\int_{-\infty}^{z_{G}} d z^{\prime}\left[\rho_{l}-\rho\left(z^{\prime}\right)\right]$ 


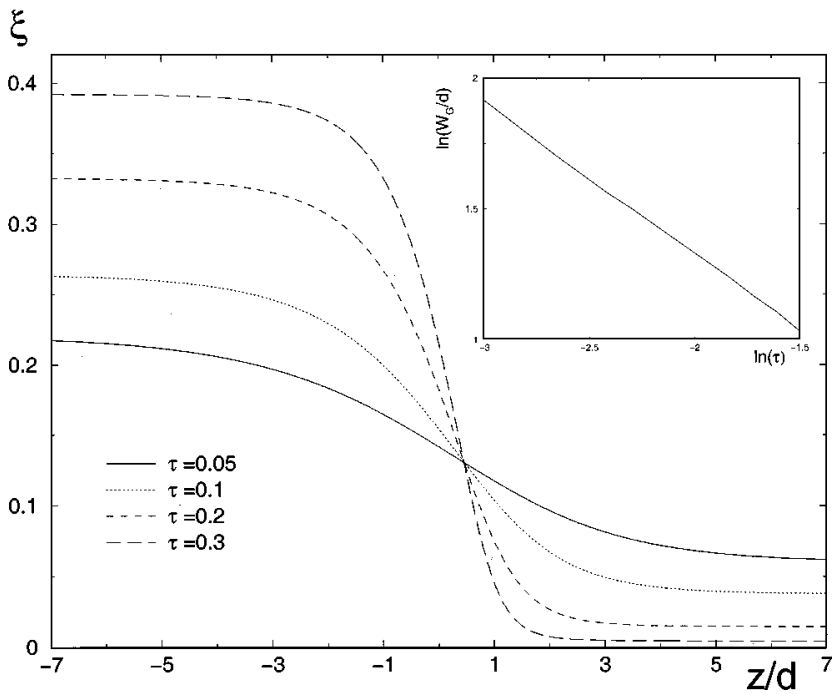

FIG. 5. The density profile $\xi(z)$ for various given $\tau$ and $Q^{*}=0.8$.

$=\int_{z_{G}}^{+\infty} d z^{\prime}\left[\rho\left(z^{\prime}\right)-\rho_{v}\right]$. In the inset of Fig. 5, we plot $\ln \left(W_{G} / d\right)$ vs $\ln \tau$ from which we obtain the scaling relation for $\tau \ll 1$,

$$
W_{G} \sim \tau^{-1 / 2} \text {. }
$$

In Fig. 6 we show the effect of temperature on the orientation order parameter $\eta_{2}(z)$ at a fixed $Q^{*}$. As the temperature increases, the profile becomes more broaden and flat. In the inset of Fig. 6 we show that the interfacial ordering scales with $\tau$ when $\tau \ll 1$ as

$$
\max \eta_{2}-\min \eta_{2} \sim \tau^{3 / 2} \text {. }
$$

Quadrupolar fluids are known to have an electric potential jump $\Delta \varphi$ across the planar liquid-vapor interface. This potential jump, given by

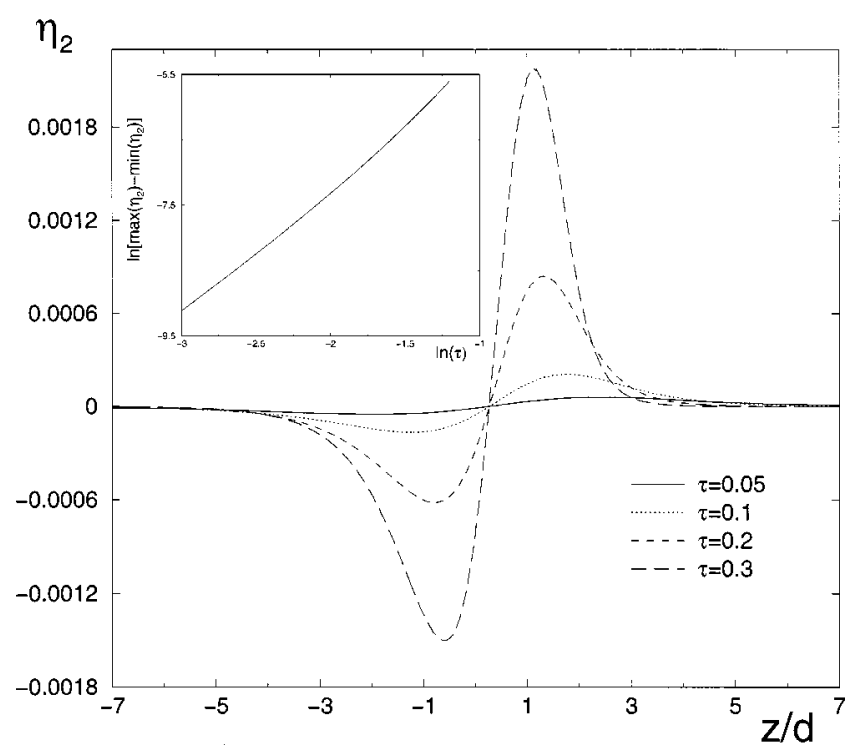

FIG. 6. The order-parameter profile $\eta_{2}(z)$ for various given $\tau$ and $Q^{*}$ $=0.8$. The inset shows that the magnitude of the orientational order $\max \eta_{2}-\min \eta_{2}$ scales as $\tau^{3 / 2}$.

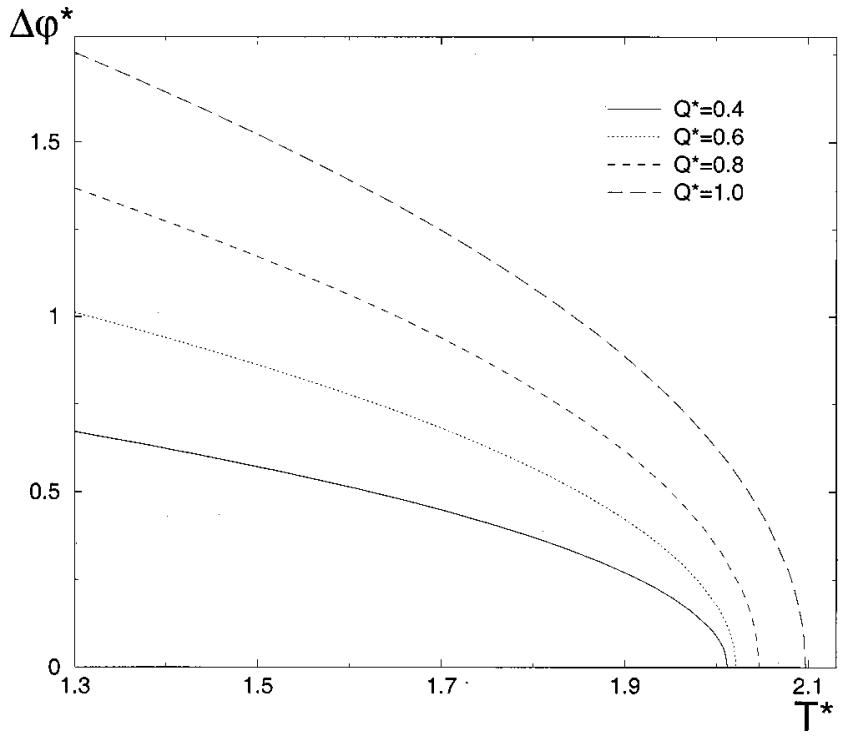

FIG. 7. Temperature ( $\left.T^{*}\right)$ dependence of the reduced surface potential $\Delta \varphi^{*}$ for various given $Q^{*}$.

$$
\Delta \varphi=\frac{2 \pi}{3} Q\left(\rho_{l}-\rho_{v}\right),
$$

is independent of the characteristics of the interface and solely dependent on bulk-phase properties and strength of the molecular quadrupole moment. ${ }^{42}$ In Fig. 7 we show the reduced surface potential $\Delta \varphi^{*}\left(\equiv \Delta \varphi(d / \epsilon)^{1 / 2}\right)$ as a function of the temperature $T^{*}$ for various given $Q^{*}$. Since $\rho_{l}-\rho_{v}$ follows the scaling relation Eq. (21), Eq. (25) immediately yields for $\tau \ll 1$,

$$
\Delta \varphi^{*} \sim \tau^{1 / 2},
$$

that is, the surface potential follows the same scaling relation as the coexisting density difference. Moreover, because $\xi_{l}$ $-\xi_{v}$ is almost independent of $Q^{*}$, it follows from Eq. (25) that at a given $\dot{\tau}$,

$$
\Delta \varphi^{*} \sim Q^{*} .
$$

We are aware of only one computer simulation ${ }^{43}$ which provides a calculation of the surface potential for quadrupolar fluid. Brodskaya and Zakharov studied a cluster of $64 \mathrm{~mol}-$ ecules of quadrupolar SSLJ model of bromine at one temperature. Because the critical temperature of the model fluid is unknown, it is not possible to compare their simulation result with the scaling relation (26) obtained here.

Finally, we calculate the thermodynamical surface tension $\sigma$, defined as the excess of the grand canonical potential per unit area,

$$
\sigma=\left(\Omega-\Omega^{\text {bulk }}\right) / A .
$$

The reduced surface tension $\sigma^{*}=\sigma\left(d^{2} / \epsilon\right)$. In Fig. 8, the temperature dependence of $\sigma^{*}$ at various given $Q^{*}$ is shown. The effect of $Q^{*}$ on $\sigma^{*}$ is similar to that of dipole moment on $\sigma^{*}$, that is, the surface tension $\sigma^{*}$ increases with the strength of quadrupole moment at a fixed temperature. ${ }^{24,26}$ This interfacial behavior was also reported by Haile et al. ${ }^{18}$ based on the Padé perturbation study of quadrupolar SSLJ fluids (see Fig. 2 in Ref. 18). In Fig. 9, we plot $\sigma^{*}$ vs the 


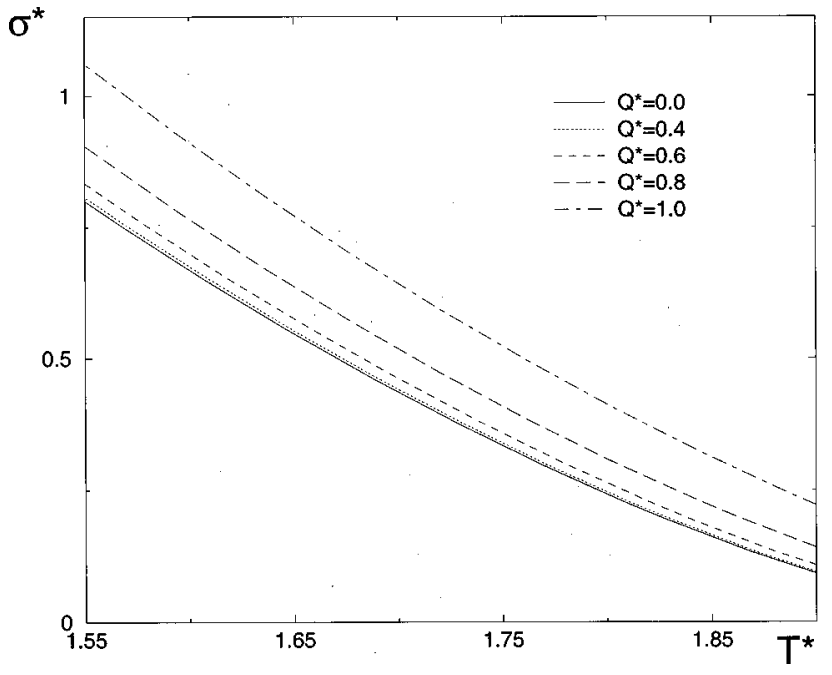

FIG. 8. Temperature $\left(T^{*}\right)$ dependence of the reduced surface tension $\sigma^{*}$ for various given $Q^{*}$.

scaled temperature $\tau$. It shows that the surface tension $\sigma^{*}$ is nearly independent of $Q^{*}$ as $\tau \longrightarrow 0$ (or $T \rightarrow T_{c}$ ). For dipolar fluids it has been found by Teixeira and Telo da Gama, ${ }^{24}$ that the surface tension $\sigma^{*}$ is nearly independent of $\mu_{0}^{*}$ for all the range of the considered scaled temperature. The inset of Fig. 9 shows the scaling relation of $\sigma^{*}(\tau)$, i.e.,

$$
\sigma^{*} \sim \tau^{3 / 2} \text {. }
$$

\section{CONCLUSION}

We have extended the modified mean-field density functional theory of Teixeira and Telo da Gama ${ }^{24}$ to treat a model quadrupolar fluid. We demonstrate that the inclusion of the quadrupole-quadrupole terms to second order in the theory permit us to observe the influence of the quadrupole strength on both the bulk-phase and interfacial properties of the quadrupolar fluid.

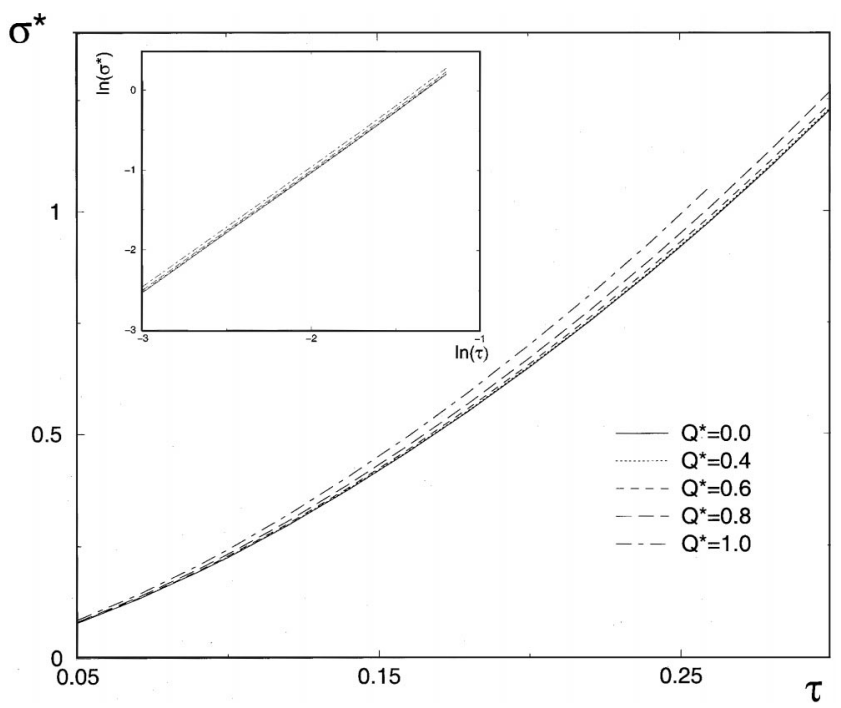

FIG. 9. Scaled temperature $(\tau)$ dependence of $\sigma^{*}$ for various given $Q^{*}$. The inset shows that $\sigma^{*}$ scales as $\tau^{3 / 2}$.
For bulk-phase properties, we find that increasing the quadrupole moment strength $Q^{*}$ leads to a higher coexisting liquid density and a lower coexisting vapor density, and raises the critical temperature $T_{c}^{*}$. For the latter, it is found $T_{c}^{*}\left(Q^{*}\right)-T_{c}^{*}(0) \sim\left(Q^{*}\right)^{4}$. It is also found that the vaporliquid coexisting densities follows the law of corresponding states. In fact, the binodal curves at various given $Q^{*}$ can be all collapsed into a single master curve. Moreover, the difference in coexisting density follows the scaling relation $\xi_{l}$ $-\xi_{v} \sim \tau^{1 / 2}$, and $\xi_{l}-\xi_{v}$ is nearly independent of $Q^{*}$.

For interfacial properties, we find based on the orientation order-parameter profile $\eta_{2}(z)$ that quadrupolar molecules at the vapor-liquid interface tend to lie parallel to the interface on the liquid side and normal to the interface on the vapor side. This orientational ordering is due entirely to the molecular quadrupole-quadrupole interaction. At a fixed temperature the orientational order, measured by the difference of the maximum and minimum value of $\eta_{2}(z)$, decreases with the quadrupole moment strength $Q^{*}$ to the fourth power $\left(Q^{*}\right)^{4}$. The orientational order also follows the scaling relation, $\max \eta_{2}(z)-\min \eta_{2}(z) \sim \tau^{3 / 2}$ at a given $Q^{*}$. It is also found that the width of density profile satisfies the scaling relation $W_{G} \sim \tau^{-1 / 2}$, and the reduced surface electric potential $\Delta \varphi^{*}$ follows the scaling relation $\Delta \varphi^{*} \sim \tau^{1 / 2}$, at fixed $Q^{*}$. Moreover, $\Delta \varphi^{*}$ is proportional to $Q^{*}$ at fixed temperature $\tau$. Finally, we find the thermodynamic surface tension $\sigma^{*}$ increases with $Q^{*}$, and at fixed $Q^{*}$ it satisfies the scaling relation $\sigma^{*} \sim \tau^{3 / 2}$.

To close, we note that purely quadrupolar fluids share many similar mean-field scaling relations as those for purely dipolar fluids. For example, Frodl and Dietrich ${ }^{26}$ have applied a nonperturbative density-functional theory to dipolar (Stockmayer) fluid. Their derived scaling relations and power-law exponents for the coexisting density difference, the magnitude of orientational order, and the surface tension are identical to ours for the quadrupolar fluid [Eqs. (20), (22)-(24), and (29)].

\section{ACKNOWLEDGMENTS}

We are grateful to Tikhon Bykov for useful discussions. This work is supported by the National Science Foundation.

\section{APPENDIX: DERIVATION OF $\phi_{\text {eff }}$ IN EQ. (9)}

Here, we derive the expression for the effective potential $\phi_{\text {eff }}$ arising in Eq. (9). Towards this end we expand the exponential function in Eq. (2) over the perturbation potential $\beta u_{\text {per }}$ with keeping the terms up to the second power of $\beta u_{\text {per }}$. Writing the radius-vector $\mathbf{r}_{12}$ in the cylindrical coordinates, i.e., $\mathbf{r}_{12}=\left(R_{12}, z_{12}, \varphi_{12}\right)$, we obtain

$$
\begin{aligned}
& \phi_{\text {eff }}\left(z_{12}, \omega_{1}, \omega_{2}\right) \\
& =\int_{0}^{2 \pi} d \varphi_{12} \int_{0}^{+\infty} R_{12} d R_{12}\left[\left(u_{\text {per }}\left(R_{12}, z_{12}, \varphi_{12}, \omega_{1}, \omega_{2}\right)\right.\right. \\
& \left.\quad-(\beta / 2) u_{\mathrm{per}}^{2}\left(R_{12}, z_{12}, \varphi_{12}, \omega_{1}, \omega_{2}\right)\right] .
\end{aligned}
$$

Substituting Eq. (5) into Eq. (A1) $\phi_{\text {eff }}$ can be expressed as 


$$
\phi_{\mathrm{eff}}\left(z_{12}, \omega_{1}, \omega_{2}\right)=\overline{u_{00}}+\overline{u_{q q}}-\frac{\beta}{2}\left[\overline{u_{00}^{2}}+2 \overline{u_{00} u_{q q}}+\overline{u_{q q}^{2}}\right] .
$$

On the right-hand side of Eq. (A2) all five terms can be expressed as

$$
\begin{aligned}
\bar{\chi}\left(z_{12}, \omega_{1}, \omega_{2}\right) & \\
= & \int_{0}^{2 \pi} d \varphi_{12} \int_{0}^{+\infty} R_{12} d R_{12} \chi\left(R_{12}, z_{12}, \varphi_{12}, \omega_{1}, \omega_{2}\right) \\
& \times H\left(r_{12}-d\right),
\end{aligned}
$$

where $\chi=u_{00}, u_{q q}, u_{00}^{2}, u_{00} u_{q q}, u_{q q}^{2}$. The integral $\overline{u_{00}}, \overline{u_{00}^{2}}$, and $\overline{u_{q q}}$ are given in Ref. 24 as

$$
\overline{u_{00}}=A(z), \quad \overline{u_{00}^{2}}=C(z), \quad \overline{u_{q q}}=\varphi_{q q}(z) P_{2} P_{2}^{\prime},
$$

where $P_{2}=P_{2}\left(\cos \theta_{1}\right), P_{2}^{\prime}=P_{2}\left(\cos \theta_{2}\right)$, and detailed expression for $A(z), C(z), \varphi_{q q}(z)$, is provided in Eq. (A14) of Ref. 24 [note that in Eq. (A4) those terms involving $\cos \left(n\left(\varphi_{1}-\varphi_{2}\right)\right)$ are removed because the orientation distribution $\hat{f}$ does not depend on $\varphi$ so that the integration over $\varphi$ for those terms will vanish].

The two remaining terms in Eq. (A2), $\overline{u_{00} u_{q q}}$ and $\overline{u_{q q}^{2}}$, are our main focus, and both include the quadrupolequadrupole interaction. First, we write the potential between two linear quadrupoles as a sum of spherical harmonics $Y_{l m},{ }^{36}$

$$
\begin{aligned}
u_{q q}= & \frac{Q^{2}}{r_{12}^{5}} \frac{8 \pi}{15}(70 \pi)^{1 / 2} \sum_{m_{1}, m_{2}, m_{3}} C\left(224 ; m_{1}, m_{2}, m_{3}\right) \\
& \times Y_{2 m_{1}}\left(\omega_{1}\right) Y_{2 m_{2}}\left(\omega_{2}\right) Y_{4 m_{3}}^{*}\left(\omega_{12}\right),
\end{aligned}
$$

where $\left|m_{1}\right|,\left|m_{2}\right| \leqslant 2, m_{3}=m_{1}+m_{2}, C\left(224 ; m_{1}, m_{2}, m_{3}\right)$ is the Clebsch-Gordan coefficient in the Rose convention, ${ }^{44}$ and $\omega_{12}$ denotes the orientation of the intermolecular axis in the space-fixed coordinate system. We then have

$$
\begin{aligned}
\overline{u_{00} u_{q q}}= & -\epsilon d^{6} Q^{2} \frac{32 \pi}{15}(70 \pi)^{1 / 2} \\
& \times \sum_{m_{1}, m_{2}, m_{3}} C\left(224 ; m_{1}, m_{2}, m_{3}\right) Y_{2 m_{1}}\left(\omega_{1}\right) \\
& \times Y_{2 m_{2}}\left(\omega_{2}\right) \cdot \int_{0}^{+\infty} \frac{R_{12} d R_{12}}{r_{12}^{11}} H\left(r_{12}-d\right) \\
& \times \int_{0}^{2 \pi} Y_{4 m_{3}}^{*}\left(\omega_{12}\right) d \varphi_{12} .
\end{aligned}
$$

With account of the expression for the spherical harmonics $Y_{l m}(\theta, \varphi)$

$$
=(-1)^{m}\left(\frac{(2 l+1)}{4 \pi}\right)^{1 / 2}\left(\frac{(l-m) !}{(l+m) !}\right)^{1 / 2} P_{l}^{m}(\cos \theta) e^{i m \varphi},
$$

where $P_{l}^{m}(x)$ is the associated Legendre polynomial, and the orthogonal relation $\int_{0}^{2 \pi} e^{i\left(m-m^{\prime}\right) \varphi} d \varphi=2 \pi \delta_{m m^{\prime}}$, Eq. (A6) becomes

$$
\begin{aligned}
\overline{u_{00} u_{q q}}= & -\epsilon d^{6} Q^{2}(8 \pi)^{2}\left(\frac{7}{10}\right)^{1 / 2} \\
& \times \sum_{m_{1}+m_{2}=0} C\left(224 ; m_{1}, m_{2}, 0\right) Y_{2 m_{1}}\left(\omega_{1}\right) Y_{2 m_{2}}\left(\omega_{2}\right) \\
& \times \int_{0}^{+\infty} \frac{R_{12} d R_{12}}{r_{12}^{11}} H\left(r_{12}-d\right) P_{4}\left(\cos \theta_{12}\right)
\end{aligned}
$$

The integral in Eq. (A7) involves $P_{4}\left(\cos \theta_{12}\right)$ which is a polynomial of $\cos \theta_{12}=z_{12} / r_{12}$ of the order four. Thus, it can be reduced to a sum of integral $\int_{0}^{+\infty} R_{12} d R_{12} / r_{12}^{n} H\left(r_{12}\right.$ $-d$ ), where $n$ is an integer greater than 2. With $r_{12}$ $=\sqrt{R_{12}^{2}+z_{12}^{2}}$, we obtain

$$
\begin{aligned}
& \int_{0}^{+\infty} \frac{R_{12} d R_{12}}{r_{12}^{n}} H\left(r_{12}-d\right) \\
& \quad=\frac{1}{(n-2)} \begin{cases}\frac{1}{d^{n-2}}, & \left|z_{12}\right| \leqslant d \\
\frac{1}{\left|z_{12}\right|^{n-2}}, & \left|z_{12}\right|>d .\end{cases}
\end{aligned}
$$

Using Eq. (A8) in Eq. (A7) we obtain

$$
\overline{u_{00} u_{q q}}=\chi_{00, q q}^{22} P_{2} P_{2}^{\prime},
$$

where

$$
\chi_{00, q q}^{22}=-\epsilon \frac{\pi Q^{2}}{d^{3}} 6\left\{\begin{array}{l}
\frac{35}{13} \frac{z^{4}}{d^{4}}-\frac{30}{11} \frac{z^{2}}{d^{2}}+\frac{1}{3}, \quad|z| \leqslant d \\
\frac{128}{429} \frac{d^{9}}{\left|z^{9}\right|}, \quad|z|>d .
\end{array}\right.
$$

We also have

$$
\begin{aligned}
& \overline{u_{q q}^{2}}=\left(\frac{8 \pi}{15}\right)^{2} Q^{4} \sum_{\substack{m_{1}, m_{2}, m_{3} \\
m_{1}^{\prime}, m_{2}^{\prime}, m_{3}^{\prime}}} C\left(224 ; m_{1}, m_{2}, m_{3}\right) \\
& \times C\left(224 ; m_{1}^{\prime}, m_{2}^{\prime}, m_{3}^{\prime}\right) Y_{2 m_{1}}\left(\omega_{1}\right) Y_{2 m_{2}}\left(\omega_{2}\right) \\
& \times Y_{2 m_{1}^{\prime}}\left(\omega_{1}\right) Y_{2 m_{2}^{\prime}}\left(\omega_{2}\right) \int_{0}^{+\infty} \frac{R_{12} d R_{12}}{r_{12}^{10}} H\left(r_{12}-d\right) \\
& \times \int d \varphi_{12} Y_{4 m_{3}}^{*}\left(\omega_{12}\right) Y_{4 m_{3}^{\prime}}^{*}\left(\omega_{12}\right) .
\end{aligned}
$$

The angular integral over $\varphi_{12}$ in Eq. (A11) is proportional to $\delta_{m_{3}, m_{3}^{\prime}}$. Therefore, 


$$
\begin{aligned}
\overline{u_{q q}^{2}}= & \left(\frac{8 \pi}{15}\right)^{2} Q^{4} \sum_{\substack{m_{1}, m_{2}, m_{3} \\
m_{1}^{\prime}, m_{2}^{\prime}}} C\left(224 ; m_{1}, m_{2}, m_{3}\right) \\
& \times C\left(224 ; m_{1}^{\prime}, m_{2}^{\prime},-m_{3}\right) Y_{2 m_{1}}\left(\omega_{1}\right) Y_{2 m_{2}}\left(\omega_{2}\right) Y_{2 m_{1}^{\prime}}\left(\omega_{1}\right) \\
& \times Y_{2 m_{2}^{\prime}}\left(\omega_{2}\right) \int_{0}^{+\infty} \frac{R_{12} d R_{12}}{r_{12}^{10}} H\left(r_{12}-d\right) P_{4}^{m_{3}} \\
& \times\left(\cos \theta_{12}\right) P_{4}^{-m_{3}}\left(\cos \theta_{12}\right),
\end{aligned}
$$

where $P_{4}^{m_{3}}\left(\cos \theta_{12}\right) P_{4}^{-m_{3}}\left(\cos \theta_{12}\right)$ is a polynomial of $\cos \theta_{12}$ with the highest power being eight. Taking the integration on $R_{12}$ for every $m_{3}$ in Eq. (A12) with using Eq. (A8), and rewriting the result as an expansion of the Legendre polynomials, we obtain, after a lengthy calculation,

$$
\begin{aligned}
\overline{u_{q q}^{2}}\left(z, \omega_{1}, \omega_{2}\right)= & \chi_{q q, q q}^{00}+\chi_{q q, q q}^{22} P_{2} P_{2}^{\prime}+\chi_{q q, q q}^{44} P_{4} P_{4}^{\prime} \\
& +\chi_{q q, q q}^{02}\left(P_{2}+P_{2}^{\prime}\right)+\chi_{q q, q q}^{04}\left(P_{4}+P_{4}^{\prime}\right) \\
& +\chi_{q q, q q}^{24}\left(P_{2} P_{4}^{\prime}+P_{2}^{\prime} P_{4}\right),
\end{aligned}
$$

where $P_{i}=P_{i}\left(\cos \theta_{1}\right), P_{i}^{\prime}=P_{i}\left(\cos \theta_{2}\right)$ and the coefficients,

$$
\begin{aligned}
& \chi_{q q, q q}^{00}=\frac{\pi Q^{4}}{d^{8}} \frac{7}{10} \begin{cases}1, & |z| \leqslant d \\
\frac{d^{8}}{z^{8}}, & |z|>d,\end{cases} \\
& \chi_{q q, q q}^{22}=\frac{\pi Q^{4}}{d^{8}} \frac{5}{49}\left\{\begin{array}{l}
27 \frac{z^{4}}{d^{4}}-\frac{156}{5} \frac{z^{2}}{d^{2}}+\frac{77}{10}, \quad|z| \leqslant d \\
\frac{7}{2} \frac{d^{8}}{z^{8}}, \quad|z|>d
\end{array}\right. \\
& \chi_{q q, q q}^{44}=\frac{\pi Q^{4}}{d^{8}} \frac{9}{784}\left\{\begin{array}{l}
\frac{60025}{8} \frac{z^{8}}{d^{8}}-16100 \frac{z^{6}}{d^{6}}+10929 \frac{z^{4}}{d^{4}}-\frac{12092}{5} \frac{z^{2}}{d^{2}}+\frac{1757}{20}, \quad|z| \leqslant d \\
\frac{63}{40} \frac{d^{8}}{z^{8}}, \quad|z|>d,
\end{array}\right. \\
& \chi_{q q, q q}^{02}=\frac{\pi Q^{4}}{d^{8}} \frac{5}{14}\left\{\begin{array}{l}
\frac{12}{5} \frac{z^{2}}{d^{2}}-1, \quad|z| \leqslant d \\
\frac{7}{5} \frac{d^{8}}{z^{8}}, \quad|z|>d,
\end{array}\right. \\
& \chi_{q q, q q}^{04}=\frac{\pi Q^{4}}{d^{8}} \frac{3}{112}\left\{\begin{array}{l}
42 \frac{z^{4}}{d^{4}}-\frac{216}{5} \frac{z^{2}}{d^{2}}+\frac{27}{5}, \quad|z| \leqslant d \\
\frac{21}{5} \frac{d^{8}}{z^{8}}, \quad|z|>d
\end{array}\right. \\
& \chi_{q q, q q}^{24}=\frac{\pi Q^{4}}{d^{8}} \frac{15}{784}\left\{\begin{array}{l}
420 \frac{z^{6}}{d^{6}}-690 \frac{z^{4}}{d^{4}}+\frac{1476}{5} \frac{z^{2}}{d^{2}}-21, \quad|z| \leqslant d \\
\frac{21}{5} \frac{d^{8}}{z^{8}}, \quad|z|>d .
\end{array}\right.
\end{aligned}
$$

Finally, to express $\phi_{\text {eff }}$ as an expansion of the Legendre polynomials we substitute Eqs. (A4), (A10), and (A13) into Eq. (A2), and obtain

$$
\begin{aligned}
\phi_{\mathrm{eff}}\left(z, \omega_{1}, \omega_{2}\right) & \\
= & \phi_{00}(z)+\phi_{22}(z) P_{2} P_{2}^{\prime}+\phi_{44}(z) P_{4} P_{4}^{\prime} \\
& +\phi_{02}(z)\left(P_{2}+P_{2}^{\prime}\right)+\phi_{04}(z)\left(P_{4}+P_{4}^{\prime}\right) \\
& +\phi_{24}(z)\left(P_{2} P_{4}^{\prime}+P_{2}^{\prime} P_{4}\right),
\end{aligned}
$$

where

$$
\begin{aligned}
& \phi_{00}(z)=A(z)-\frac{\beta}{2}\left(C(z)+\chi_{q q, q q}^{00}(z)\right), \\
& \phi_{22}(z)=\varphi_{q q}(z)-\frac{\beta}{2}\left(2 \chi_{00, q q}^{22}(z)+\chi_{q q, q q}^{22}(z)\right), \\
& \phi_{44}(z)=-\frac{\beta}{2} \chi_{q q, q q}^{44}(z), \quad \phi_{02}(z)=-\frac{\beta}{2} \chi_{q q, q q}^{02}(z), \\
& \phi_{04}(z)=-\frac{\beta}{2} \chi_{q q, q q}^{04}(z), \quad \phi_{24}(z)=-\frac{\beta}{2} \chi_{q q, q q}^{24}(z) .
\end{aligned}
$$


${ }^{1}$ D. E. Stogrin and A. P. Stogrin, Mol. Phys. 11, 371 (1966).

${ }^{2}$ K. S. Shing and K. E. Gubbins, Mol. Phys. 45, 129 (1982).

${ }^{3}$ M. R. Stapleton, D. J. Tildesley, A. Z. Panagiotopoulos, and N. Quirke, Mol. Simul. 2, 147 (1989).

${ }^{4}$ B. Smit and C. P. Williams, J. Phys.: Condens. Matter 2, 4281 (1990).

${ }^{5}$ B. Garzon, S. Lado, C. Vega, E. de Miguel, and L. F. Rull, J. Chem. Phys. 101, 4166 (1994).

${ }^{6}$ J. Winkelmann, Fluid Phase Equilib. 48, 67 (1989).

${ }^{7}$ S. F. O'Shea, G. S. Dubey, and J. C. Rasaiah, J. Chem. Phys. 107, 237 (1997).

${ }^{8}$ M. Lombardero, C. Martin, E. Lomba, and A. Fernandez, Fluid Phase Equilib. 74, 95 (1992).

${ }^{9}$ B. Saager and J. Fischer, Fluid Phase Equilib. 72, 67 (1992).

${ }^{10}$ L. L. Lee, E. Assad, H. A. Kwong, T. H. Chung, and J. M. Haile, Physica A 110, 235 (1982)

${ }^{11}$ C. Martin, M. Lombardero, and E. Lomba, J. Chem. Phys. 98, 6465 (1993).

${ }^{12}$ S. Gupta, J. Ram, and R. C. Singh, Physica A 278, 447 (2000).

${ }^{13}$ B. Garzon, S. Lago, and C. Vega, Mol. Phys. 96, 123 (1999).

${ }^{14}$ C. Vega and K. Gubbins, Mol. Phys. 72, 881 (1992).

${ }^{15}$ M. C. Wojcik and K. E. Gubbins, J. Phys. Chem. 88, 6559 (1984).

${ }^{16}$ R. Pospisil, A. Malijevsky, and S. Labik, Mol. Phys. 68, 609 (1989).

${ }^{17}$ T. Boublik, Mol. Phys. 77, 983 (1992).

${ }^{18}$ J. M. Haile, C. G. Gray, and K. E. Gubbins, J. Chem. Phys. 64, 2569 (1976).

${ }^{19}$ S. M. Thompson, K. E. Gubbins, and J. M. Haile, J. Chem. Phys. 75, 1325 (1981).

${ }^{20}$ S. M. Thompson and K. E. Gubbins, J. Chem. Phys. 74, 6467 (1981).

${ }^{21}$ J. Eggebrecht, K. E. Gubbins, and S. M. Thompson, J. Chem. Phys. 86, 2286 (1987).

${ }^{22}$ M. Kasch and F. Forstmann, J. Chem. Phys. 99, 3037 (1993).

${ }^{23}$ S. Iatzevich and F. Forstmann, Mol. Phys. 98, 1309 (2000).

${ }^{24}$ P. I. Teixeira and M. M. Telo da Gama, J. Phys.: Condens. Matter 3, 111 (1991).
${ }^{25}$ P. Frodl and S. Dietrich, Phys. Rev. A 45, 7330 (1992).

${ }^{26}$ P. Frodl and S. Dietrich, Phys. Rev. E 48, 3741 (1993).

${ }^{27}$ P. I. Teixeira, B. S. Almeida, M. M. Telo da Gama, J. A. Rueda, and R. G. Rubio, J. Phys. Chem. 96, 8488 (1992).

${ }^{28}$ B. Yang, D. E. Sullivan, B. Tjipto-Margo, and C. G. Grey, Mol. Phys. 76, 709 (1992).

${ }^{29}$ V. Talanquer and D. W. Oxtoby, J. Chem. Phys. 99, 4670 (1993).

${ }^{30}$ V. B. Warshavsky, T. V. Bykov, and X. C. Zeng, J. Chem. Phys. 114, 504 (2001).

${ }^{31}$ T. V. Bykov and X. C. Zeng, J. Phys. Chem. B 105, 11586 (2001).

${ }^{32}$ E. Chacón, P. Tarazona, and G. Navascues, J. Chem. Phys. 79, 4426 (1983).

${ }^{33}$ N. E. Carnahan and K. E. Starling, J. Chem. Phys. 51, 635 (1969).

${ }^{34}$ T. M. Reed and K. E. Gubbins, Applied Statistical Mechanics (McGrawHill, New York, 1973), p. 117.

${ }^{35}$ J. O. Hirschfelder, C. F. Curtis, and R. B. Bird, Molecular Theory of Gases and Liquids (Wiley, New York, 1954), pp. 549-551.

${ }^{36}$ G. Gray and K. E. Gubbins, The Theory of Molecular Fluids (Clarendon, Oxford, 1984).

${ }^{37}$ B. Smit, Ph. de Smedt, and D. Frenkel, Mol. Phys. 68, 973 (1989).

${ }^{38}$ B. Smit, C. P. Williams, E. M. Hendriks, and S. W. de Leeuw, Mol. Phys. 68, 765 (1989).

${ }^{39}$ G. S. Dubey and S. F. O'Shea, Phys. Rev. E 49, 2175 (1994).

${ }^{40}$ J. Eggebrecht, S. M. Thompson, and K. E. Gubbins, J. Chem. Phys. 86, 2299 (1987)

${ }^{41}$ J. S. Rowlinson and B. Widom, Molecular Theory of Capillarity (Claredon, Oxford, 1982).

${ }^{42}$ M. A. Wilson, A. Pohorille, and L. R. Pratt, J. Chem. Phys. 90, 5211 (1989).

${ }^{43}$ E. N. Brodskaya and V. V. Zakharov, J. Chem. Phys. 102, 4595 (1995).

${ }^{44}$ M. E. Rose, Elementary Theory of Angular Momentum (Wiley, New York, 1957). 